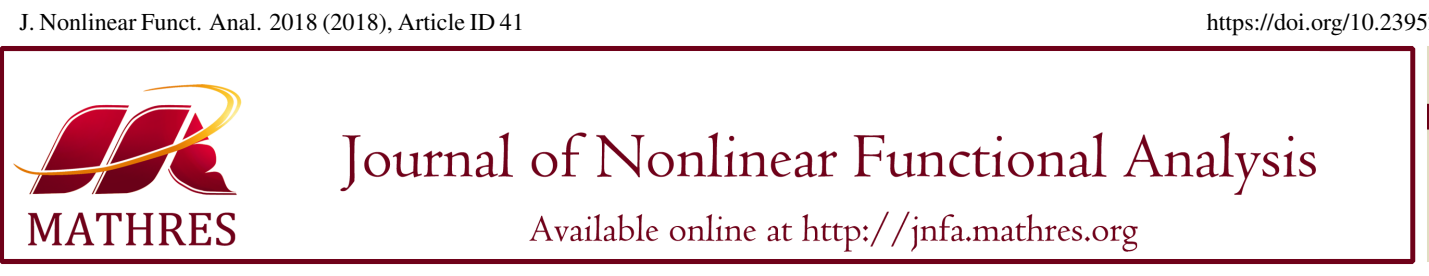

\title{
OPTIMIZATION OF THE BOLZA PROBLEM WITH SECOND ORDER DIFFERENTIAL INEQUALITIES
}

\author{
ELIMHAN N. MAHMUDOV ${ }^{1,2, *}$, IZZET GÖKSEL ${ }^{1}$ \\ ${ }^{1}$ Department of Mathematics, Istanbul Technical University, Turkey \\ ${ }^{2}$ Institute of Control Systems, Azerbaijan National Academy of Sciences, Azerbaijan
}

\begin{abstract}
This paper concerns optimization of the Bolza problem with convex and nonconvex second order discrete and differential state variable inequality constraints. Necessary and sufficient conditions of optimality for second order discrete and differential inequalities are derived. According to proposed discretization method, the problem with discrete-approximation inequalities is investigated. Equivalence theorems for subdifferential inclusions are basic tools in the study of optimality conditions for continuous problems. This approach plays a much more important role in the derivation of second order adjoint discrete and differential inequality constraints generated by given inequality constraints. A numerical example is presented to illustrate the theoretical result.
\end{abstract}

Keywords. Bolza problem; Differential inequality; Differential inclusion; Discrete-approximation problem.

2010 Mathematics Subject Classification. 34A60, 49J15.

\section{INTRODUCTION-PRELIMINARIES}

Optimization problems with inequality constraints have wide applications in economy, engineering design, industrial optimization, game theory and so on. Many authors are interested in studying this kind of problems and have made great achievements. Especially, differential inequalities appear naturally in several areas of applied mathematics, such as, in mathematical economics in problems of resource allocation and in the study of planning procedures, in mechanics in the study of elastoplastic systems and in differential games. Constraint optimization, which has many real word applications, is therefore of paramount importance in several areas of mathematics and other sciences. Many mathematical problems in literature can be formulated as variational inequality problems with continuous multivalued mappings and their relationships with other general problems of nonlinear and variational analysis; see [1]-[11] and the references therein.

In this paper, we discuss a special kind of optimization problem, in which the constraints are defined by second order discrete and differential nonlinear state variable inequality constraints (SVICs).

${ }^{*}$ Corresponding author.

E-mail addresses: elimhan22@yahoo.com (E.N. Mahmudov), gokseli@itu.edu.tr (İ. Göksel).

Received July 8, 2018; Accepted November 25, 2018.

(c)2018 Journal of Nonlinear Functional Analysis 
One general approach to the SVIC optimization relies on the representation of the sublevel sets of the inequality constraints via set-valued mappings. This analysis reveals the hidden relationship between optimal control methods with inequality constraints and set-valued mappings. The difficulty in the problems with higher order differential inclusions (or differential inequalities) is rather to construct the EulerLagrange type higher order adjoint inclusions and the suitable boundary conditions. That is why only the qualitative properties of second order differential inclusions (inequalities) are investigated recently, see, for examples, $[1,4,11,12]$ and references therein. In [12], the existence of viable solutions for an autonomous second order functional differential inclusion was proved in the case that multifunction defined the inclusion, which is upper semicontinuous compact valued and contained in the subdifferential of a proper lower semicontinuous convex function. The investigated optimization problem is the logical continuation of the paper [13], which is mainly concerned with the necessary and sufficient conditions of the optimality for the Bolza problem with higher order discrete and differential inclusions. Applying optimality conditions of problems with geometric constraints for arbitrary higher order (say $s$-order) discrete inclusions, optimality conditions are formulated. Also, some special transversality conditions, which are peculiar to problems including third order derivatives, are formulated. In the first part of paper [14], optimization of polyhedral discrete and differential inclusions was considered. This problem was reduced to a convex minimization problem and the necessary and sufficient condition for optimality was derived. In particular, boundedness of the set of adjoint discrete solutions and upper semi-continuity of the locally adjoint mapping were proved. In the second part, an optimization problem described by convex inequality constraints was studied. By using the equivalence theorem concerning the subdifferential calculus and approximating method, a necessary and sufficient condition for the discrete-approximation problem with inequality constraint was established. In [15], the author studied a new class of problems of optimal control theory with Sturm-Liouville type differential inclusions involving second order linear self-adjoint differential operators. By using the discretization method guaranteeing transition to the continuous problem, the discrete and discrete-approximate inclusions were investigated. Necessary and sufficient conditions, containing both the Euler-Lagrangian and Hamiltonian type inclusions and transversality conditions were derived. For the Mayer problem in its general form, the chain rule for subdifferentiation of composition of convex functions was used. In [16], the necessary conditions of optimality were derived for optimal control problems with pathwise state constraints, in which the dynamic constraint was modelled as a differential inclusion. Note that the existing results devoted to constraint optimal control problems deal with the usual state constraints of zero order (see [16] and references therein), i.e., when the inequality constraints do not involve the derivative of a feasible trajectory. The stated problems and obtained optimality conditions in our paper are new. Novelty of the problem formulation is justified. The paper can be divided into three parts. In the first part of this paper, we formulate an optimal control problem, in which the system dynamics are described by so-called second order discrete SVICs. In the second part, an optimization problem with inequality constraints involving first and second order derivatives of a sought function is formulated, i.e., second order differential SVICs. The last part is devoted to the construction of the discrete-approximation problem for second order differential SVICs and on the basic results for second order discrete SVICs, we formulate sufficient conditions of optimality for differential SVICs.

In Section 1, the necessary notions and results such as the local tent, convex upper approximation (CUA) of non-smooth functions, etc., are given for the readers convenience from Mahmudov's book [17] 
and papers $[13,15,18]$. In Section 2, Cauchy problems for second order discrete and differential SVICs are formulated. In Section 3, the optimality problem for posed second order discrete SVICs are reduced to the problem with finite number of geometric constraints. By constructions of convex and nonsmooth analysis, necessary and sufficient conditions of optimality for second order SVICs are proved. Under nondegeneracy assumptions imposed on the optimal trajectory for a convex problem, necessary and sufficient conditions are proved. As a consequence, under the existence of convex upper approximation and local tents, the optimality conditions for nonconvex second order SVICs are obtained. In Section 4, by using first and second order difference operators with the problem for second order differential SVICs, we associate the second order discrete-approximation problem with SVICs. It seems that the transition to the discrete-approximation problem requires some special equivalence theorems, which allow us to make a bridge between the main results of second order discrete and discrete-approximation problems with SVICs. By using local tents and CUAs, similar results are shown for nonconvex problems. Obviously, this method can also play an important role in numerical procedures. In Section 5, we derive sufficient conditions of the optimality for the second order discrete SVICs. Formulation of these conditions is based on the limit procedure in the optimality conditions for the second order differential SVIC. By applying the second order adjoint discrete SVICs generated by inequality constraints and the discrete approximations method, we derive sufficient optimality conditions for the second order differential SVICs. Usually, the second order adjoint differential SVICs involve some auxiliary adjoint variables.

In what follows, the necessary standard notions can be found in $[13,15,17,18]$. As usual, $\mathbb{R}^{n}$ is the $n$-dimensional Euclidean space, $\langle x, v\rangle$ is the inner product of the elements $x, v \in \mathbb{R}^{n}$ and $(x, v)$ is a pair of $x, v$. By int $A$ and $r i A$, we denote the interior and relative interior of a set $A \subset \mathbb{R}^{3 n}$, respectively.

Definition 1.1. [15] A convex cone $K_{A}\left(z_{0}\right), z=\left(x, v_{1}, v_{2}\right)$ is called the cone of tangent directions at a point $z_{0}=\left(x^{0}, v_{1}^{0}, v_{2}^{0}\right) \in A$ if it follows from $\bar{z}=\left(\bar{x}, \bar{v}_{1}, \bar{v}_{2}\right) \in K_{A}\left(z_{0}\right)$ that $\bar{z}$ is a tangent vector to the set $A$, i.e., there exists a function $\phi(\lambda) \in \mathbb{R}^{3 n}$ satisfying $z_{0}+\lambda \bar{z}+\phi(\lambda) \in A$ for sufficiently small $\lambda>0$, where $\lambda^{-1} \phi(\lambda) \rightarrow 0$ as $\lambda \downarrow 0$.

Clearly, for a convex set $A$ at a point $\left(x^{0}, v_{1}^{0}, v_{2}^{0}\right) \in A$, setting $\phi(\lambda) \equiv 0$, we have $K_{A}\left(z_{0}\right)=\{(\bar{x}, \bar{u}, \bar{v})$ : $\left.\bar{x}=\lambda\left(x_{1}-x^{0}\right), \bar{u}=\lambda\left(u_{1}-v_{1}^{0}\right), \bar{v}=\lambda\left(v_{1}-v_{2}^{0}\right), \lambda>0\right\}, \forall\left(x, u_{1}, v_{1}\right) \in A$.

$K_{A}{ }^{*}\left(x^{0}, v_{1}^{0}, v_{2}^{0}\right)$ is the dual of the cone of tangent vectors $K_{A}\left(x^{0}, v_{1}^{0}, v_{2}^{0}\right)$.

Definition 1.2. [17] For an arbitrary nonempty subset $P \subset \mathbb{R}^{3 n}$, the cone defined by

$$
\text { cone } P=\left\{\left(\bar{x}, \bar{v}_{1}, \bar{v}_{2}\right): \bar{x}=\alpha x, \bar{v}_{1}=\alpha v_{1}, \bar{v}_{2}=\alpha v_{2},\left(x, v_{1}, v_{2}\right) \in P, \alpha>0\right\}
$$

is called the cone generated by $P$.

By Definition 1.1, we see that the cone of tangent directions involves directions for each of which there exists a function $\phi(\lambda)$. But in order to predetermine the properties of set $A$, this is not sufficient. Nevertheless, the following notion of a local tent allows us to predetermine a mapping in $A$ for the nearest tangent directions among themselves.

Definition 1.3. The cone $K_{A}\left(z_{0}\right)$ is called the local tent if for any $\overline{z_{0}} \in$ ri $K_{A}\left(z_{0}\right)$, there exists a convex cone $K \subseteq K_{A}\left(z_{0}\right)$ and a continuous mapping $\gamma(\bar{z})$ defined in the neighbourhood of the origin such that

(1) $\overline{z_{0}} \in$ ri $K$, $\operatorname{Lin} K=\operatorname{Lin} K_{A}\left(z_{0}\right)$, where $\operatorname{Lin} K$ is the linear span of $K$,

(2) $\gamma(\bar{z})=\bar{z}+r(\bar{z}), r(\bar{z})\|\bar{z}\|^{-1} \rightarrow 0$ as $\bar{z} \rightarrow 0$, 
(3) $z_{0}+\gamma(\bar{z}) \in A, \bar{z} \in K \cap S_{\varepsilon}(0)$ for some $\varepsilon>0$, where $S_{\varepsilon}(0)$ is the ball of radius $\varepsilon$. [15]

Definition 1.4. $[17,18] h(\bar{z}, z)$ is called a convex upper approximation (CUA) of the function $\Omega: \mathbb{R}^{3 n} \rightarrow$ $\mathbb{R}^{1} \cup\{ \pm \infty\}$ at a point $z \in \operatorname{dom} \Omega=\{z:|\Omega(z)|<+\infty\}$ if

(1) $h(\bar{z}, z) \geq V(\bar{z}, z), \forall \bar{z} \neq 0$,

(2) $h(\bar{z}, z)$ is a convex closed (or lower semicontinuous) positive homogeneous function in $\bar{z}$, where

$$
V(\bar{z}, z)=\sup _{r(\cdot)} \limsup _{\lambda \downarrow 0} \frac{1}{\lambda}[\Omega(z+\lambda \bar{z}+r(\lambda))-\Omega(z)], \lambda^{-1} r(\lambda) \rightarrow 0 .
$$

Here, the exterior supremum is taken on all $r(\lambda)$ such that $\lambda^{-1} r(\lambda) \rightarrow 0$ as $\lambda \downarrow 0$.

Definition 1.5. [15, 17] The set defined as

$$
\partial h(0, z)=\left\{z^{*} \in \mathbb{R}^{3 n}: h(\bar{z}, z) \geq\langle\bar{z}, z\rangle, \bar{z} \in \mathbb{R}^{3 n}\right\}
$$

is called a subdifferential of the function $\Omega$ at a point $z$ and is denoted by $\partial \Omega(z)$.

The main advantage of this definition is its simplicity. It is known [17] that in the convexity of $\Omega$, the given definition coincides with the usual definition of a subdifferential. It should be noted that, for various classes of functions, the notion of subdifferential can be defined in different ways. The first concept of subdifferential for general nonconvex functions was introduced by Clarke (see e.g. [5] and references therein) who performed pioneering work in the area of nonsmooth analysis spread far beyond the scope of convexity. In particular, Clarke developed a comprehensive subdifferential calculus for his generalized gradients of locally Lipschitzian functions defined on Banach spaces.

The Mordukhovichs subdifferential consists of a main class of generalized differentials and plays a vital role in pure and applied analysis. We refer readers to book volumes [19] for key issues about these subdifferentials from theoretical and applied points of view in the modern variational analysis. A function $\Omega$ is called a proper function if it does not assume the value $-\infty$ and is not identically equal to $+\infty$.

\section{Problem Statement}

In this section, we formulate the problems with the so-called second order discrete and differential SVICs.

At the beginning, we deal with the following second order discrete model labelled as (PD):

$$
\begin{aligned}
& \operatorname{minimize} \sum_{t=2}^{N-1} f\left(x_{t}, t\right), \\
& \Omega\left(x_{t}, x_{t+1}, x_{t+2}\right) \leq 0, t=0, \ldots, N-2, \\
& x_{0}=\bar{v}_{0}, x_{1}=\bar{v}_{1},
\end{aligned}
$$

where $x_{t} \in \mathbb{R}^{n}, f(\cdot, t)(t=0, \ldots, N-2)$ are real-valued continuously differentiable convex functions, $f(\cdot, t): \mathbb{R}^{n} \rightarrow \mathbb{R}^{1} \cup\{ \pm \infty\}, \Omega: \mathbb{R}^{n} \rightarrow \mathbb{R}^{1}$ and $f(\cdot, N-1)$ are continuous convex functions, $N$ is a fixed natural number, and $\bar{v}_{1}, \bar{v}_{2}$ are fixed vectors.

In fact, the conditions in (2.3) are discrete analogs of the Cauchy initial conditions for second order discrete SVICs. A sequence $\left\{x_{t}\right\}_{t=0}^{N}=\left\{x_{t}: t=0,1, \ldots, N\right\}$ is called a feasible trajectory of the stated problem in (2.1)-(2.3). The question is to find a feasible trajectory of the problem in (2.1)-(2.3) that 
minimizes $\sum_{t=2}^{N-1} f\left(x_{t}, t\right)$. The problem in (2.1)-(2.3) is convex if $\Omega$ and $f(\cdot, N-1)$ are continuous convex functions and $f(\cdot, t)(t=2, \ldots, N-2)$ is a continuously differentiable proper convex function.

Condition $H_{1}$ : Suppose that in the convex problem (PD) for point $x_{t}^{0} \in \mathbb{R}^{n}$, one of the following cases is fulfilled:

(1) $\left(x_{t}^{0}, x_{t+1}^{0}, x_{t+2}^{0}\right) \in \operatorname{ri} F, x_{t}^{0} \in \operatorname{ri}(\operatorname{dom} f(\cdot, t)), t=0, \ldots, N-2$

(2) $\left(x_{t}^{0}, x_{t+1}^{0}, x_{t+2}^{0}\right) \in$ int $F, F=\left\{\left(x, v_{1}, v_{2}\right): \Omega\left(x, v_{1}, v_{2}\right) \leq 0\right\}$ (with the possible exception of one fixed $\left.t_{0}\right)$ and $f(\cdot, t)$ are continuous at $x_{t}^{0}$.

In what follows, this condition will be called the nondegeneracy condition.

Condition $\mathrm{H}_{2}$ : Assume that in the nonconvex case of the problem (PD), the cones of tangent directions $K_{F}\left(\tilde{x}_{t}, \tilde{x}_{t+1}, \tilde{x}_{t+2}\right)$ are local tents, where $\tilde{x}_{t}$ are the points of the optimal trajectory $\left\{\tilde{x}_{t}\right\}_{t=0}^{N}$. Suppose, moreover, that both functions $\Omega\left(x_{t}, x_{t+1}, x_{t+2}\right)$ and $f(\cdot, N-1)$ admit continuous convex upper approximations (CUAs) $h_{\Omega}^{t}\left(\cdot, \tilde{x}_{t}, \tilde{x}_{t+1}, \tilde{x}_{t+2}\right)$ and $h_{N-1}\left(\cdot, \tilde{x}_{N-1}\right)$, respectively [18] which ensures that the subdifferential$\mathrm{s} \partial_{\left(x, v_{1}, v_{2}\right)} \Omega\left(\tilde{x}_{t}, \tilde{x}_{t+1}, \tilde{x}_{t+2}\right)=\partial h_{\Omega}^{t}\left(0, \tilde{x}_{t}, \tilde{x}_{t+1}, \tilde{x}_{t+2}\right)$ and $\partial f\left(\tilde{x}_{N-1}, N-1\right)=\partial h_{N-1}\left(0, \tilde{x}_{N-1}\right)$ are defined. Here, it is assumed that $\Omega\left(\tilde{x}_{t}, \tilde{x}_{t+1}, \tilde{x}_{t+2}\right)=0$. Furthermore, let $f(\cdot, t)(t=2, \ldots, N-2)$ be continuously differentiable nonconvex functions, i.e., $h_{t}\left(\bar{x}, \tilde{x}_{t}\right)=\left\langle\bar{x}, f^{\prime}\left(\tilde{x}_{t}, t\right)\right\rangle$; see [17].

Besides, if $\Omega$ is a continuously differentiable nonconvex function, then the cone $K_{F}\left(z_{0}\right), z_{0} \in F$ defined as

$$
K_{F}\left(z_{0}\right)=\left\{\bar{z}:\left\langle\bar{z}, \Omega_{z}^{\prime}\left(z_{0}\right)\right\rangle<0\right\}
$$

is a local tent.

It should be noted that, for a convex set $F \subset \mathbb{R}^{3 n}$, the cone defined as follows is a local tent and always exists [17]:

$$
K_{F}\left(z_{0}\right)=\left\{\bar{z}: \bar{z}=\lambda\left(z_{1}-z\right), \lambda>0, \forall z_{1} \in F\right\} .
$$

Section (5) deals with the following problem for second order differential SVICs, labelled as (PC):

$$
\begin{aligned}
& \operatorname{minimize} J[x(\cdot)]=\int_{0}^{1} f(x(t), t) d t+q(x(1)), \\
& \Omega\left(x(t), x^{\prime}(t), x^{\prime \prime}(t)\right) \leq 0, \text { a.e. } t \in[0,1], \\
& x_{0}=v_{0}, x^{\prime}(0)=v_{1},
\end{aligned}
$$

where at first for simplicity, the convex problem is considered; $f$ is continuously differentiable and convex with respect to $x, \Omega$ and $q$ are continuous convex functions and $v_{0}, v_{1}$ are fixed vectors. (Note that in Theorem (5.5), the obtained result for the convex problem is extended in a more general nonconvex setting of Problem (PC).) It is required to find an arc $\tilde{x}(t)$ of the Cauchy problem given by (2.1)-(2.3) for the second order SVICs satisfying the inequality in (2.5) almost everywhere (a.e.) on $[0,1]$ and the initial conditions in (2.6) that minimize the Bolza functional $J[x(\cdot)]$. For this purpose, we first derive necessary optimality conditions for the discrete-approximation problem and then, by passing to the limit procedure, we establish sufficient optimality conditions to the Problem (PC) described by second order differential SVICs. Here, a feasible trajectory $x(\cdot)$ is understood to be an absolutely continuous twice differentiable function on $[0,1]$ for which $x^{\prime \prime}(\cdot) \in L_{1}^{n}([0,1])$. Notice that such class of functions is a Banach space, endowed with different equivalent norms. 


\section{Optimality CONDitions for SECond Order Discrete SVICS}

First, we consider the convex problem (PD). After transforming (PD) to an equivalent form by using a convex programming method and subdifferential calculus, we derive an optimality condition for it.

Let us define the following convex sets in space $\mathbb{R}^{n(N+1)}$

$$
\begin{gathered}
M_{t}=\left\{w=\left(x_{0}, \ldots, x_{N}\right): \Omega\left(x_{t}, x_{t+1}, x_{t+2}\right) \leq 0\right\}, t=0,1, \ldots, N-2, \\
G_{0}=\left\{w=\left(x_{0}, \ldots, x_{N}\right): x_{0}=\bar{v}_{0}\right\}, \quad G_{1}=\left\{w=\left(x_{0}, \ldots, x_{N}\right): x_{1}=\bar{v}_{1}\right\},
\end{gathered}
$$

where $w=\left(x_{0}, x_{1}, \ldots, x_{N}\right) \in \mathbb{R}^{n(N+1)}$.

By denoting $g(w)=\sum_{t=2}^{N-1} f\left(x_{t}, t\right)$, we can replace the convex problem (PD) by the equivalent problem with geometric constraints:

$$
\text { minimize } g(w) \text { subject to } Q=G_{0} \cap G_{1} \cap\left(\bigcap_{t=0}^{N-2} M_{t}\right) \text {. }
$$

Let us compute the dual cone $K_{M_{t}}^{*}(w)$ generated by the subdifferential set $\partial_{z} \Omega\left(x_{t}, x_{t+1}, x_{t+2}\right)$, where $K_{M_{t}}(w)$ is a cone of tangent directions.

Proposition 3.1. Let cone $\partial_{z} \Omega\left(x_{t}, x_{t+1}, x_{t+2}\right), z=\left(x, v_{1}, v_{2}\right)$ be the cone generated by the subdifferential set $\partial_{z} \Omega\left(x_{t}, x_{t+1}, x_{t+2}\right)$. Then, under the nondegeneracy condition, we have

$K_{M_{t}}^{*}(w)=\left\{w^{*}=\left(x_{0}^{*}, \ldots, x_{N}^{*}\right):\left(x_{t}^{*}, x_{t+1}^{*}, x_{t+2}^{*}\right) \in\left[-\right.\right.$ cone $\left.\left.\partial_{z} \Omega\left(x_{t}, x_{t+1}, x_{t+2}\right)\right], x_{k}^{*}=0, k \neq t, t+1, t+2\right\}$.

Proof. Obviously, by Definition (1.1) if $w+\lambda \bar{w} \in M_{t}, t=0, \ldots, N-2$ for sufficiently small $\lambda>0$, i.e., $\left(x_{t}+\lambda \bar{x}_{t}, x_{t+1}+\lambda \bar{x}_{t+1}, x_{t+2}+\lambda \bar{x}_{t+2}\right) \in F, F=\left\{\left(x, v_{1}, v_{2}\right): \Omega\left(x, v_{1}, v_{2}\right) \leq 0\right\}$, then it follows that $\bar{w} \in K_{M_{t}}(w)$. So

$$
\begin{gathered}
K_{M_{t}}(w)=\left\{\bar{w}:\left(\bar{x}_{t}, \bar{x}_{t+1}, \bar{x}_{t+2}\right) \in K_{F}\left(x_{t}, x_{t+1}, x_{t+2}\right)\right\}, \\
K_{F}\left(x_{t}, x_{t+1}, x_{t+2}\right) \equiv \text { cone }\left[F-\left(x_{t}, x_{t+1}, x_{t+2}\right)\right] .
\end{gathered}
$$

Now, we recall that by Theorem 1.34 in [17] that

$$
K_{F}^{*}\left(x_{t}, x_{t+1}, x_{t+2}\right)= \begin{cases}\{0\} \in \mathbb{R}^{3 n}, & \text { if } \Omega\left(x_{t}, x_{t+1}, x_{t+2}\right)<0, \\ - \text { cone } \partial_{\left(x, v_{1}, v_{2}\right)} \Omega\left(x_{t}, x_{t+1}, x_{t+2}\right), & \text { if } \Omega\left(x_{t}, x_{t+1}, x_{t+2}\right)=0 .\end{cases}
$$

Then, the proof of the proposition follows immediately from the arbitrariness of components $x_{k}, k \neq$ $t, t+1, t+2$ of vectors $\bar{w}$. This completes the proof.

It is not hard to derive the following formulas:

$$
\begin{aligned}
& K_{G_{0}}^{*}(w)=\left\{w^{*}=\left(x_{0}^{*}, \ldots, x_{N}^{*}\right): x_{t}^{*}=0, t \neq 0\right\}, \\
& K_{G_{1}}^{*}(w)=\left\{w^{*}=\left(x_{0}^{*}, \ldots, x_{N}^{*}\right): x_{t}^{*}=0, t \neq 1\right\} .
\end{aligned}
$$

In the sense of the cone generated by the subdifferential set $\partial_{z} \Omega\left(x_{t}, x_{t+1}, x_{t+2}\right)$, we are ready to prove the optimality conditions for the problem (PD).

Theorem 3.2. Let $\Omega$ be a continuous convex function. Let $f(\cdot, t)(t=2, \ldots, N-2)$ be a continuously differentiable proper convex function in $x$, and let $f(\cdot, N-1)$ be a continuous convex proper function. Let $f(\cdot, t)$ be continuous at points $x_{t}$ of some feasible trajectory. Then, for optimality of the trajectory $\left\{\tilde{x}_{t}\right\}_{t=0}^{N}$ in the problem (PD), it is necessary that there exist numbers $\mu \in\{0,1\}, \alpha_{t} \geq 0, t=0, \ldots, N-2$ 
and vectors $x_{t}^{*}, x_{N}^{*}, u_{t}^{*}, t=0, \ldots, N-1$ simultaneously not all equal to zero satisfying the second order adjoint discrete SVICs generated by the inequality constraints given in (2.2)

$$
\begin{gathered}
\left(x_{t}^{*}-u_{t}^{*}-\mu f_{x}^{\prime}\left(\tilde{x}_{t}, t\right), u_{t+1}^{*},-x_{t+2}^{*}\right) \in \alpha_{t} \partial_{\left(x, v_{1}, v_{2}\right)} \Omega\left(\tilde{x}_{t}, \tilde{x}_{t+1}, \tilde{x}_{t+2}\right), \\
\alpha_{t} \partial_{\left(x, v_{1}, v_{2}\right)} \Omega\left(\tilde{x}_{t}, \tilde{x}_{t+1}, \tilde{x}_{t+2}\right)=0, \alpha_{t} \geq 0, t=0,1, \ldots, N-2, \quad u_{0}^{*}=0, \quad f_{x}^{\prime}\left(\tilde{x}_{0}, 0\right)=f_{x}^{\prime}\left(\tilde{x}_{1}, 1\right)=0
\end{gathered}
$$

and boundary conditions

$$
x_{N-1}^{*}-u_{N-1}^{*} \in \mu \partial f\left(\tilde{x}_{N-1}, N-1\right), x_{N}^{*}=0 .
$$

In addition, if the nondegeneracy condition is satisfied, then these conditions are sufficient for optimality of the trajectory of $\left\{\tilde{x}_{t}\right\}_{t=0}^{N}$.

Proof. Clearly, if $\left\{\tilde{x}_{t}\right\}_{t=0}^{N}$ is an optimal trajectory, we claim that $\tilde{w}=\left(\tilde{x}_{0}, \ldots, \tilde{x}_{N}\right)$ is a solution of the convex mathematical programming problem (3.1). Then, it is known (see [17, 19]) that there exist not all zero vectors $w^{*}(t) \in K_{M_{t}}^{*}(\tilde{w}), t=0,1, \ldots, N-2, w_{0}^{*} \in K_{G_{0}}^{*}(\tilde{w}), w_{1}^{*} \in K_{G_{1}}^{*}(\tilde{w})$ and a number $\mu \in\{0,1\}$ such that

$$
\mu w^{0 *}=\sum_{t=0}^{N-2} w^{*}(t)+w_{0}^{*}+w_{1}^{*}, \quad w^{0 *} \in \partial g(\tilde{w}) .
$$

From the definition of function $g$, we claim that the vector $w^{0 *}$ is of the form $w^{0 *}=\left(x_{00}^{*}, x_{10}^{*}, \ldots, x_{N 0}^{*}\right)$, $x_{t 0}^{*}=f_{x}^{\prime}\left(\tilde{x}_{t}, t\right)(t=0, \ldots, N-2), x_{(N-1) 0}^{*} \in \partial_{x} f\left(\tilde{x}_{N-1}, N-1\right)$. By Proposition 3.1 and formulas in (3.2), it can be easily seen that

$$
\begin{aligned}
& w^{*}(t)=\left(0, \ldots, 0, x_{t}^{*}(t), x_{t+1}^{*}(t), x_{t+1}^{*}(t), 0, \ldots, 0\right), \\
& \left(-x_{t}^{*}(t),-x_{t+1}^{*}(t),-x_{t+2}^{*}(t)\right) \in \operatorname{cone} \partial_{\left(x, v_{1}, v_{2}\right)} \Omega\left(\tilde{x}_{t}, \tilde{x}_{t+1}, \tilde{x}_{t+2}\right), t=2, \ldots, N-2, \\
& w_{0}^{*}=\left(\bar{x}^{*}, 0, \ldots, 0\right), \\
& w_{1}^{*}=\left(0, \overline{\bar{x}}^{*}, 0, \ldots, 0\right),
\end{aligned}
$$

where $\bar{x}^{*}, \overline{\bar{x}}^{*}$ are arbitrary vectors. Besides, from the component-wise representation of (3.3), we have

$$
\begin{aligned}
& \mu x_{t 0}^{*}=x_{t}^{*}(t)+x_{t}^{*}(t-1)+x_{t}^{*}(t-2), t=2, \ldots, N-2, \\
& \bar{x}^{*}+x_{0}^{*}(0)=0, \\
& \overline{\bar{x}}^{*}+x_{1}^{*}(1)+x_{1}^{*}(0)=0 .
\end{aligned}
$$

Note that

$$
\begin{gathered}
\text { cone } \partial_{\left(x, v_{1}, v_{2}\right)} \Omega\left(x_{t}, x_{t+1}, x_{t+2}\right)=\left\{\left(\alpha_{t} \hat{x}_{t}^{*}, \alpha_{t} \hat{x}_{t+1}^{*}, \alpha_{t} \hat{x}_{t+2}^{*}\right):\left(\hat{x}_{t}^{*}, \hat{x}_{t+1}^{*}, \hat{x}_{t+2}^{*}\right) \in \partial_{\left(x, v_{1}, v_{2}\right)} \Omega\left(x_{t}, x_{t+1}, x_{t+2}\right),\right. \\
\left.\alpha_{t} \Omega\left(x_{t}, x_{t+1}, x_{t+2}\right)=0, \alpha_{t} \geq 0\right\} .
\end{gathered}
$$

From the second formula of (3.4), we conclude that

$$
\begin{aligned}
& -x_{t}^{*}(t)=\alpha_{t} \hat{x}_{t}^{*},-x_{t+1}^{*}(t)=\alpha_{t} \hat{x}_{t+1}^{*},-x_{t+2}^{*}(t)=\alpha_{t} \hat{x}_{t+2}^{*}, t=2, \ldots, N-2, \\
& \left(\hat{x}_{t}^{*}, \hat{x}_{t+1}^{*}, \hat{x}_{t+2}^{*}\right) \in \partial_{\left(x, v_{1}, v_{2}\right)} \Omega\left(\tilde{x}_{t}, \tilde{x}_{t+1}, \tilde{x}_{t+2}\right), \alpha_{t} \Omega\left(\tilde{x}_{t}, \tilde{x}_{t+1}, \tilde{x}_{t+2}\right)=0, \alpha_{t} \geq 0 .
\end{aligned}
$$

Note $\alpha_{t} \hat{x}_{t}^{*}=-x_{t}^{*}(t), \alpha_{t} \hat{x}_{t+1}^{*}=-x_{t+1}^{*}(t), \alpha_{t} \hat{x}_{t+2}^{*}=-x_{t+2}^{*}(t)$. By the second formula of (3.6), it follows that

$$
\begin{aligned}
& \left(-x_{t}^{*}(t),-x_{t+1}^{*}(t),-x_{t+2}^{*}(t)\right) \in \alpha_{t} \partial_{\left(x, v_{1}, v_{2}\right)} \Omega\left(\tilde{x}_{t}, \tilde{x}_{t+1}, \tilde{x}_{t+2}\right), \\
& \alpha_{t} \Omega\left(\tilde{x}_{t}, \tilde{x}_{t+1}, \tilde{x}_{t+2}\right)=0, \alpha_{t} \geq 0, t=2, \ldots, N-2 .
\end{aligned}
$$


Now, by introducing the new notations $x_{t+1}^{*}(t) \equiv-u_{t+1}^{*}, x_{t+2}^{*}(t) \equiv x_{t+2}^{*}, t=1, \ldots, N-2$ in the first equation in (3.5), we derive from (3.7) that

$$
\begin{aligned}
& \left(x_{t}^{*}-u_{t}^{*}-\mu x_{t 0}^{*}, u_{t+1}^{*},-x_{t+2}^{*}\right) \in \alpha_{t} \partial_{\left(x, v_{1}, v_{2}\right)} \Omega\left(\tilde{x}_{t}, \tilde{x}_{t+1}, \tilde{x}_{t+2}\right), \\
& \alpha_{t} \Omega\left(\tilde{x}_{t}, \tilde{x}_{t+1}, \tilde{x}_{t+2}\right)=0, \alpha_{t} \geq 0, t=2, \ldots, N-2 .
\end{aligned}
$$

Moreover, it is easy to see that by setting $f\left(x_{0}, 0\right)=f\left(x_{1}, 1\right)=0, \bar{x}^{*}=-x_{0}^{*}, u_{0}^{*} \equiv 0$ and $\bar{x}^{*}=-x_{1}^{*}$ in the second and third equalities, respectively, we can generalize the formula in (3.8) to the case $t=0,1$. Consequently, for $t=N-1$, we have

$$
\mu x_{(N-1) 0}^{*}=x_{N-1}^{*}-u_{N-1}^{*} .
$$

Since $f\left(\tilde{x}_{N}, N\right) \equiv 0$, it follows that $x_{N}^{*}=0$. Thus, taking into account the formulas (3.8) and (3.9), we deduce that the indicated conditions of the theorem are necessary for optimality.

On the other hand, it is not hard to see from Theorem 3.3 in [18] that under the nondegeneracy condition, the equality in (3.3) holds with parameter $\mu=1$, which completes the sufficiency.

Below, we show how the result of Theorem (3.2) can be extended to the problem (PD) in the nonconvex case.

Theorem 3.3. Assume that condition $H_{2}$ for the nonconvex problem (PD) is satisfied; $\Omega$ and $f(\cdot, N-1)$ admit continuous CUAs $h_{\Omega}^{t}\left(\cdot, \tilde{x}_{t}, \tilde{x}_{t+1}, \tilde{x}_{t+2}\right)$ and $h_{N-1}\left(\cdot, \tilde{x}_{N-1}\right)$, respectively, i.e., $\partial_{\left(x, v_{1}, v_{2}\right)} \Omega\left(\tilde{x}_{t}, \tilde{x}_{t+1}, \tilde{x}_{t+2}\right)$ $=\partial h_{\Omega}^{t}\left(0, \tilde{x}_{t}, \tilde{x}_{t+1}, \tilde{x}_{t+2}\right)$ and $\partial f\left(\tilde{x}_{N-1}, N-1\right)=\partial h_{N-1}\left(0, \tilde{x}_{N-1}\right), f(\cdot, t)(t=2, \ldots, N-2)$ are continuously differentiable nonconvex functions. In addition, let $\bar{z}$ be a point such that $h_{\Omega}^{t}\left(\bar{z}, \tilde{x}_{t}, \tilde{x}_{t+1}, \tilde{x}_{t+2}\right)<0$ and $\Omega\left(\tilde{x}_{t}, \tilde{x}_{t+1}, \tilde{x}_{t+2}\right)=0$. Then, for optimality of the trajectory $\left\{\tilde{x}_{t}\right\}_{t=0}^{N}$ in the nonconvex problem with the second order discrete SVICs, it is necessary that there exist numbers $\mu \geq 0, \alpha_{t} \geq 0, t=2, \ldots, N-2$ and a pair of vectors $\left\{x_{t}^{*}\right\},\left\{u_{t}^{*}\right\}$ not all equal to zero, satisfying the analogous conditions in the previous case.

Proof. In the nonconvex problem (PD), the assumption $\mathrm{H}_{2}$ ensures the conditions of Theorem 3.24 in [17] for the equivalent problem (3.1). Then, by analogy with this theorem for the basic relation (3.3) and completely by the similar way, we derive the necessary condition as in Theorem (3.2). Note that the required cone

$$
K_{F}\left(\tilde{x}_{t}, \tilde{x}_{t+1}, \tilde{x}_{t+2}\right)=\left\{\bar{z}: h_{\Omega}^{t}\left(\bar{z}, \tilde{x}_{t}, \tilde{x}_{t+1}, \tilde{x}_{t+2}\right)<0\right\}
$$

is a local tent.

Remark 3.4. Because of the arbitrariness of $\bar{x}^{*}, \bar{x}^{*}\left(\bar{x}^{*}=-x_{0}^{*}, \bar{x}^{*}=-x_{1}^{*}\right)$, it is not hard to see that the second and third equalities of formulas (3.5) are always realized. Then, it is clear that, without loss of generality, the conditions $u_{0}^{*}=0, f_{x}^{\prime}\left(\tilde{x}_{0}, 0\right)=f_{x}^{\prime}\left(\tilde{x}_{1}, 1\right)=0$ can be neglected and second order discrete adjoint SVICs are preserved for $t=2,3, \ldots, N-2$.

\section{Equivalence of Subdifferentials and Optimization of Discrete-Approximation Problems With SECOND ORder Discrete SVICS}

First, let us recall Theorem 3.1 in [13] which will be crucial in what follows. By this theorem, for the proper convex function $\Phi\left(x, v_{1}, v_{2}\right) \equiv \Omega\left[x, \frac{1}{\delta}\left(v_{1}-x\right), \frac{1}{\delta^{2}}\left(v_{2}-2 v_{1}+x\right)\right]$, the following inclusions are 
equivalent:

$$
\begin{aligned}
& \text { (1) }\left(\bar{x}^{*}, \bar{v}_{1}^{*}, \bar{v}_{2}^{*}\right) \in \partial_{\left(x, v_{1}, v_{2}\right)} \Phi\left(z_{0}\right), z_{0}=\left(x^{0}, v_{1}^{0}, v_{2}^{0}\right) \in \operatorname{dom} \Phi \\
& \text { (2) }\left(\bar{x}^{*}+\bar{v}_{1}^{*}+\bar{v}_{2}^{*}, \delta \bar{v}_{1}^{*}+2 \delta \bar{v}_{2}^{*}, \delta^{2} \bar{v}_{2}^{*}\right) \in \partial \Omega\left[x^{0}, \frac{1}{\delta}\left(v_{1}^{0}-x^{0}\right), \frac{1}{\delta^{2}}\left(v_{2}^{0}-2 v_{1}^{0}+x^{0}\right)\right] .
\end{aligned}
$$

Proposition 4.1. For a proper convex function defined by $\Phi\left(x, v_{1}, v_{2}\right) \equiv \Omega_{1}\left[x, \frac{1}{\delta^{2}}\left(v_{2}-2 v_{1}+x\right)\right]$, the following inclusions under the condition that $\bar{v}_{1}^{*}=-2 \bar{v}_{2}^{*}$ are equivalent:

$$
\begin{aligned}
& \text { (1) }\left(\bar{x}^{*}, \bar{v}_{1}^{*}, \bar{v}_{2}^{*}\right) \in \partial_{z} \Phi\left(z_{0}\right), z_{0}=\left(x^{0}, v_{1}^{0}, v_{2}^{0}\right) \in \operatorname{dom} \Phi, \\
& \text { (2) }\left(\bar{x}^{*}-\bar{v}_{2}^{*}, \delta^{2} \bar{v}_{2}^{*}\right) \in \partial \Omega_{1}\left[x^{0}, \frac{1}{\delta^{2}}\left(v_{2}^{0}-2 v_{1}^{0}+x^{0}\right)\right] .
\end{aligned}
$$

Proof. Note that $\partial_{z} \Phi\left(z_{0}\right)$ is a convex closed set and is bounded for $z_{0} \in \operatorname{ri}(\operatorname{dom} \Phi)$ (see $\left.[18,17,10]\right)$. Furthermore, by definition of the subdifferential, one has

$$
\begin{gathered}
\partial_{z} \Phi\left(z_{0}\right)=\left\{\left(\bar{x}^{*}, \bar{v}_{1}^{*}, \bar{v}_{2}^{*}\right): \Phi(z)-\Phi\left(z_{0}\right) \geq\left\langle\bar{x}^{*}, x-x^{0}\right\rangle+\left\langle\bar{v}_{1}^{*}, v_{1}-v_{1}^{0}\right\rangle+\left\langle\bar{v}_{2}^{*}, v_{2}-v_{2}^{0}\right\rangle,\right. \\
\left.\forall z=\left(x, v_{1}, v_{2}\right) \in \mathbb{R}^{3 n}\right\} .
\end{gathered}
$$

Similarly, one has

$$
\begin{gathered}
\partial \Omega_{1}\left[x^{0}, \frac{1}{\delta^{2}}\left(v_{2}^{0}-2 v_{1}^{0}+x^{0}\right)\right]=\left\{\left(x^{*}, v^{*}\right): \Omega_{1}\left[x, \frac{1}{\delta^{2}}\left(v_{2}-2 v_{1}+x\right)\right]-\Omega_{1}\left[x^{0}, \frac{1}{\delta^{2}}\left(v_{2}^{0}-2 v_{1}^{0}+x^{0}\right)\right]\right. \\
\geq\left\langle x^{*}, x-x^{0}\right\rangle+\left\langle v^{*}, \frac{1}{\delta^{2}}\left(v_{2}-2 v_{1}+x\right)-\frac{1}{\delta^{2}}\left(v_{2}^{0}-2 v_{1}^{0}+x^{0}\right)\right\rangle, \\
\left.\forall z=\left(x, v_{1}, v_{2}\right) \in \mathbb{R}^{3 n}\right\} .
\end{gathered}
$$

It follows that

$$
\begin{aligned}
\partial \Omega_{1}\left[x^{0}, \frac{1}{\delta^{2}}\left(v_{2}^{0}-2 v_{1}^{0}+x^{0}\right)\right]=\{ & \left(x^{*}, v^{*}\right):\left\langle x^{*}+\frac{v_{2}^{*}}{\delta^{2}}, x-x^{0}\right\rangle+\left\langle-\frac{2 v^{*}}{\delta^{2}}, v_{1}-v_{1}^{0}\right\rangle \\
& \left.+\left\langle\frac{v^{*}}{\delta^{2}}, v_{2}-v_{2}^{0}\right\rangle, \forall z=\left(x, v_{1}, v_{2}\right) \in \mathbb{R}^{3 n}\right\} .
\end{aligned}
$$

By comparing (4.2) and (4.3), we can derive that

$$
\bar{x}^{*}=x^{*}+\frac{v^{*}}{\delta^{2}}, \quad \bar{v}_{1}^{*}=-\frac{2 v^{*}}{\delta^{2}}, \quad \bar{v}_{2}^{*}=\frac{v^{*}}{\delta^{2}},
$$

whence

$$
x^{*}=\bar{x}^{*}-\bar{v}_{2}^{*}, \quad \bar{v}_{1}^{*}=-2 \bar{v}_{2}^{*}, \quad v_{2}^{*}=\delta^{2} \bar{v}_{2}^{*} .
$$

This means that they are equivalent.

The next proposition is a simple modification of Theorem 3.1 in [13].

Proposition 4.2. For a proper convex function defined by $\Phi\left(x, v_{1}, v_{2}\right) \equiv \Omega_{2}\left[x, \frac{1}{\delta}\left(v_{1}-x\right)\right]$, the following inclusions under the condition that $\bar{v}_{2}^{*} \equiv 0$ are equivalent:

$$
\begin{aligned}
& \text { (1) }\left(\bar{x}^{*}, \bar{v}_{1}^{*}, \bar{v}_{2}^{*}\right) \in \partial_{z} \Phi\left(z_{0}\right), z_{0}=\left(x^{0}, v_{1}^{0}, v_{2}^{0}\right) \in \operatorname{dom} \Phi, \\
& \text { (2) }\left(\bar{x}^{*}+\bar{v}_{1}^{*}, \delta \bar{v}_{1}^{*}\right) \in \partial \Omega_{2}\left[x^{0}, \frac{1}{\delta}\left(v_{1}^{0}-x^{0}\right)\right] .
\end{aligned}
$$


Proof. Clearly for all $\left(x, v_{1}\right) \in \mathbb{R}^{2 n}$, we have

$$
\begin{aligned}
\partial \Omega_{2}\left[x^{0}, \frac{1}{\delta}\left(v_{1}^{0}-x^{0}\right)\right]= & \left\{\left(x^{*}, v^{*}\right): \Omega_{2}\left[x, \frac{1}{\delta}\left(v_{1}-x\right)\right]-\Omega_{2}\left[x^{0}, \frac{1}{\delta}\left(v_{1}^{0}-x^{0}\right)\right]\right. \\
& \left.\geq\left\langle x^{*}-\frac{v^{*}}{\delta}, x-x^{0}\right\rangle+\left\langle\frac{v^{*}}{\delta}, v_{1}-v_{1}^{0}\right\rangle\right\} .
\end{aligned}
$$

Then, the proof of the proposition follows immediately from relations (4.2) and (4.4).

Next, we describe an idea of constructing the discrete-approximation problem for (PC). First, we introduce the first and second order difference operators:

$$
\Delta x(t)=\frac{1}{\delta}[x(t+\delta)-x(t)], \Delta^{2} x(t)=\frac{1}{\delta}[\Delta x(t+\delta)-\Delta x(t)], t=0, \delta, \ldots, 1-2 \delta,
$$

where $\delta$ is a step on the $t$-axis and $x(t)$ is a grid function on a uniform grid on $[0,1]$.

We define the following discrete-approximation problem associated with the problem (PC):

$$
\begin{aligned}
& \operatorname{minimize} J_{\delta}[x(\cdot)]=\sum_{t=2 \delta, \ldots, 1-2 \delta} \delta f(x(t), t)+q(x(1-\delta)), \\
& \text { subject to } \Omega\left(x(t), \Delta x(t), \Delta^{2} x(t)\right) \leq 0, t=0, \delta, 2 \delta, \ldots, 1-2 \delta, \\
& \qquad x(0)=v_{0}, \Delta x(0)=v_{1} .
\end{aligned}
$$

We apply the results of Theorem (3.2) to problem (4.5). To this end, we reduce problem (4.5) to the following problem labelled as (PDA):

$$
\begin{aligned}
& \operatorname{minimize} J_{\delta}[x(\cdot)]=\sum_{t=2 \delta, \ldots, 1-2 \delta} \delta f(x(t), t)+q(x(1-\delta)) \\
& \Phi(x(t), x(t+\delta), x(t+2 \delta)) \leq 0, t=0, \delta, 2 \delta, \ldots, 1-2 \delta \\
& x(0)=v_{0}, x(\delta)=v_{0}+\delta v_{1}
\end{aligned}
$$

where

$$
\Phi(x(t), x(t+\delta), x(t+2 \delta)) \equiv \Omega\left(x(t), \Delta x(t), \Delta^{2} x(t)\right) .
$$

An immediate useful consequence of the equivalence theorems, which are the basic tools in the study of discrete-approximation problems, is the following result.

Theorem 4.3. Let $\Omega$ and $q$ be continuous convex functions, and let $f(\cdot, t)(t=2 \delta, 3 \delta, \ldots, 1-2 \delta)$ be continuously differentiable proper functions convex with respect to $x$. Besides, $f(\cdot, t)$ is assumed to be continuous at points $x_{t}$ of some feasible trajectory. Then, for optimality of the trajectory $\{\tilde{x}(t)\}$ in the discrete approximation problem (4.6)-(4.7), it is necessary that there exist numbers $\mu=\mu_{\delta} \in$ $\{0,1\}, \alpha(t) \geq 0$ and a pair of vectors $\left\{x^{*}(t), u^{*}(t)\right\}$, simultaneously not all equal to zero, satisfying the second order adjoint SVICs generated by the inequality constraints in (4.7)

$$
\begin{aligned}
& {\left[\frac{1}{\delta^{2}}\left(x^{*}(t)-u^{*}(t)+u^{*}(t+\delta)-x^{*}(t+2 \delta)-\mu \delta f_{x}^{\prime}(\tilde{x}(t), t)\right)\right.} \\
& \left.\frac{1}{\delta}\left(u^{*}(t+\delta)-2 x^{*}(t+2 \delta),-x^{*}(t+2 \delta)\right)\right] \in \alpha(t) \partial_{\left(x, v_{1}, v_{2}\right)} \Omega\left(\tilde{x}(t), \Delta \tilde{x}(t), \Delta^{2} \tilde{x}(t)\right), \\
& \alpha(t) \Omega\left(\tilde{x}(t), \Delta \tilde{x}(t), \Delta^{2} \tilde{x}(t)\right)=0, \alpha(t) \geq 0, t=2 \delta, 3 \delta, \ldots, 1-2 \delta
\end{aligned}
$$

and boundary conditions

$$
x^{*}(1-\delta)-u^{*}(1-\delta) \in \mu \partial q(\tilde{x}(1-\delta)), \quad x^{*}(1)=0 .
$$


In addition, under the nondegeneracy condition, these conditions are also sufficient for optimality of $\{\tilde{x}(t)\}$.

Proof. The proof is immediate from the results of Theorem (3.2). Indeed, by Theorem (3.2) for optimality of the trajectory $\{\tilde{x}(t)\}:=\{\tilde{x}(t): t=0, \delta, \ldots, 1\}$ in problem (4.6)-(4.7), it is necessary that there exist a pair of vectors $\left\{u^{*}(t), x^{*}(t)\right\}$ and numbers $\mu=\mu_{\delta} \in\{0,1\}, \alpha(t) \geq 0$, not all zero, such that

$$
\begin{gathered}
\left(x^{*}(t)-u^{*}(t)-\mu \delta f_{x}^{\prime}(\tilde{x}(t), t), u^{*}(t+\delta),-x^{*}(t+2 \delta)\right) \in \alpha(t) \partial_{z} \Phi(\tilde{x}(t), \tilde{x}(t+\delta), \tilde{x}(t+2 \delta)), \\
\alpha(t) \Phi(\tilde{x}(t), \tilde{x}(t+\delta), \tilde{x}(t+2 \delta))=0, \alpha(t) \geq 0, t=2 \delta, \ldots, 1-2 \delta, \\
u^{*}(0)=0, f_{x}^{\prime}(\tilde{x}(0), 0)=f_{x}^{\prime}(\tilde{x}(\delta), \delta)=0, \\
x^{*}(1-\delta)-u^{*}(1-\delta) \in \mu \partial q(\tilde{x}(1-\delta)), \quad x^{*}(1)=0 .
\end{gathered}
$$

So, it remains only to express in the relationship (4.12), the subdifferential $\partial_{z} \Phi(\tilde{x}(t), \tilde{x}(t+\delta), \tilde{x}(t+2 \delta))$ in terms of the subdifferential $\partial_{z} \Omega\left(\tilde{x}(t), \Delta \tilde{x}(t), \Delta^{2} \tilde{x}(t)\right)$. Indeed, by Theorem (3.2) and formula (4.1), the second order adjoint SVICs (4.12) for the convex problem take the form (4.10), where the values $\alpha(t) / \delta^{2}$ and $\mu / \delta$ are denoted again by $\alpha(t)$ and $\mu$, respectively.

Remark 4.4. Analogously, by using Theorem (3.3), we can generalize the result of Theorem (4.3) to the nonconvex problem (PDA). Suppose that the condition $\mathrm{H}_{2}$ is satisfied for the nonconvex problem (PDA). Then, for optimality of the trajectory $\{\tilde{x}(t)\}$, it is necessary that there exist numbers $\mu \geq 0, \alpha(t) \geq 0$ and a pair of vectors $\left\{x^{*}(t), u^{*}(t)\right\}$, simultaneously not all equal to zero, satisfying second order adjoint SVICs in (4.10) and boundary conditions in (4.11) rewritten for the nonconvex case.

Next, we shall investigate the discrete problems for different particular cases of $\Omega$ function.

Theorem 4.5. Suppose that in problem $(P C) \Omega\left(x, x^{\prime}, x^{\prime \prime}\right) \equiv \Omega_{1}\left(x, x^{\prime \prime}\right)$, i.e., the function $\Omega$ does not depend on the second argument $x^{\prime}$. Then, for the corresponding (PDA) problem, the conditions (4.10)-(4.11) have the forms

$$
\begin{aligned}
& {\left[\Delta^{2} x^{*}(t) \mu f_{x}^{\prime}(\tilde{x}(t), t),-x^{*}(t+2 \delta)\right] \in \alpha(t) \partial_{\left(x, v_{2}\right)} \Omega_{1}\left(\tilde{x}(t), \Delta^{2} \tilde{x}(t)\right), t=2 \delta, 3 \delta, \ldots, 1-2 \delta} \\
& \text { and } x^{*}(1-\delta) \in \mu \partial q(\tilde{x}(1-\delta)), x^{*}(1)=0,
\end{aligned}
$$

respectively.

Proof. By applying Proposition 4.1 to the second order adjoint SVICs in (4.12), we have

$$
\begin{aligned}
& {\left[\frac{1}{\delta^{2}}\left(x^{*}(t)-u^{*}(t)+u^{*}(t+\delta)-x^{*}(t+2 \delta)-\mu \delta f_{x}^{\prime}(\tilde{x}(t), t)\right),-x^{*}(t+2 \delta)\right]} \\
& \in \alpha(t) \partial_{\left(x, v_{2}\right)} \Omega\left(\tilde{x}(t), \Delta^{2} \tilde{x}(t)\right) .
\end{aligned}
$$

On the other hand, from Proposition 4.1, $\bar{v}_{1}^{*}=-2 \bar{v}_{2}^{*}$ which from second order adjoint SVICs in (4.12) means that $u^{*}(t)=2 x^{*}(t+\delta)$. Taking into account the last condition in the left hand side of (4.14), we have

$$
\frac{1}{\delta^{2}}\left[x^{*}(t)-u^{*}(t)+u^{*}(t+\delta)-x^{*}(t+2 \delta)\right]=\Delta^{2} x^{*}(t) .
$$

Finally, by substituting $u^{*}(1-\delta)=2 x^{*}(1)$ into (4.13), we have the desired result. This completes the proof. 
Theorem 4.6. Suppose that in problem $(P C) \Omega\left(x, x^{\prime}, x^{\prime \prime}\right) \equiv \Omega_{2}\left(x, x^{\prime}\right)$, i.e., the function $\Omega$ does not depend on the third argument $x^{\prime \prime}$. Then, for the corresponding (PDA) problem, the second order adjoint SVICs in (4.10) and boundary conditions in (4.11) have the forms

$$
\begin{aligned}
& {\left[\left(\Delta u^{*}(t)-\mu f_{x}^{\prime}(\tilde{x}(t), t), u^{*}(t+\delta)\right)\right] \in \alpha(t) \partial_{\left(x, v_{1}\right)} \Omega_{2}(\tilde{x}(t), \Delta \tilde{x}(t)), t=2 \delta, 3 \delta, \ldots, 1-2 \delta,} \\
& -u^{*}(1-\delta) \in \mu \partial q(\tilde{x}(1-\delta)), x^{*}(1)=0 .
\end{aligned}
$$

Proof. Using Proposition 4.2 and inclusion in (4.12) and taking into account that $x^{*}(1-\delta)=0\left(\bar{v}_{2}^{*} \equiv 0\right)$, we have

$$
\left[\frac{1}{\delta}\left(u^{*}(t+\delta)-u^{*}(t)-\mu \delta f_{x}^{\prime}(\tilde{x}(t), t)\right), u^{*}(t+\delta)\right] \in \alpha(t) \partial_{\left(x, v_{1}\right)} \Omega_{2}(\tilde{x}(t), \Delta \tilde{x}(t))
$$

which implies the inclusion in (4.15). Substituting $x^{*}(t) \equiv 0$ into (4.11), we have

$$
-u^{*}(1-\delta) \in \mu \partial q(\tilde{x}(1-\delta)) .
$$

This completes the proof.

Let us now rewrite (4.10) and (4.11) in a more convenient form.

Lemma 4.7. The second order adjoint SVICs in (4.10) and boundary conditions in (4.11) have the forms

$$
\begin{aligned}
& {\left[\Delta^{2} x^{*}(t)+\psi^{*}(t)-\mu f_{x}^{\prime}(\tilde{x}(t), t), \psi^{*}(t),-x^{*}(t+2 \delta)\right] \in \alpha(t) \partial \Omega\left(x(t), \Delta x(t), \Delta^{2} x(t)\right),} \\
& \text { and } \psi^{*}(1)+\Delta x^{*}(1-\delta) \in \mu \partial q(\tilde{x}(1-\delta)), x^{*}(1)=0, \\
& \psi^{*}(t)=\frac{1}{\delta}\left[u^{*}(t)-2 x^{*}(t+\delta)\right], t=2 \delta, 3 \delta, \ldots, 1-2 \delta
\end{aligned}
$$

respectively.

Proof. The condition $u^{*}(t+\delta)=\delta \psi^{*}(t+\delta)+2 x^{*}(t+2 \delta)$ immediately yields

$$
\frac{1}{\delta^{2}}\left[x^{*}(t)-u^{*}(t)+u^{*}(t+\delta)-x^{*}(t+2 \delta)\right]=\Delta^{2} x^{*}(t)+\Delta \psi^{*}(t) .
$$

Moreover, by the boundary conditions in (4.11), one has

$$
\frac{1}{\delta}\left[x^{*}(1-\delta)+2 x^{*}(1)-x^{*}(1)-u^{*}(1-\delta)\right] \in \mu \partial q(\tilde{x}(1-\delta)) .
$$

Consequently, one has

$$
-\psi^{*}(1-\delta)-\Delta x^{*}(1-\delta) \in \mu \partial q(\tilde{x}(1-\delta))
$$

Taking into account (4.17) and (4.18) in the second order adjoint SVICs in (4.10) and boundary conditions in (4.11) respectively, we have the desired result. This completes the proof.

\section{SUFFICIENT CONDITIONS OF OPTIMALITY FOR SECOND ORDER DIFFERENTIAL SVIC}

By using the obtained results in Section 4, we can formulate a sufficient condition of optimality for the continuous problem (PC). Indeed, setting $\mu=1$ in conditions of Lemma 4.7 and by passing to the limit procedure as $\delta \rightarrow 0$, we establish the so-called second order adjoint differential SVICs generated by the given differential SVICs in (2.5):

$(i)\left(\frac{d^{2} x^{*}(t)}{d t^{2}}+\frac{d \psi^{*}(t)}{d t}-f_{x}^{\prime}(\tilde{x}(t), t), \psi^{*}(t)-x^{*}(t)\right) \in \alpha(t) \partial_{\left(x, v_{1}, v_{2}\right)} \Omega\left(\tilde{x}(t), \tilde{x}^{\prime}(t), \tilde{x}^{\prime \prime}(t)\right)$, a.e. $t \in[0,1]$. 
We also have

$$
\text { (ii) } \alpha(t) \Omega\left(\tilde{x}(t), \tilde{x}^{\prime}(t), \tilde{x}^{\prime \prime}(t)\right)=0, \alpha(t) \geq 0 \text {, a.e. } t \in[0,1]
$$

and the boundary conditions

$$
(\text { iii })-\psi^{*}(1)-\frac{d x^{*}(1)}{d t} \in \partial q(\tilde{x}(1)), x^{*}(1)=0 .
$$

In what follows, we assume that $x^{*}(t), t \in[0,1]$ is an absolutely continuous, twice differentiable function on $[0,1]$ for which $x^{* \prime \prime}(\cdot) \in L_{1}^{n}([0,1])$. Besides, $\psi^{*}(t), t \in[0,1]$ is absolutely continuous and $\psi^{* \prime}(\cdot) \in L_{1}^{n}([0,1])$.

The next theorem suggests a convenient way to check that the pair of absolutely continuous functions $\left\{x^{*}(t), \psi^{*}(t)\right\}$ is optimal.

Theorem 5.1. Let $f(\cdot, t): \mathbb{R}^{n} \rightarrow \mathbb{R}^{1}$ be a continuously differentiable convex function with respect to $x$, and $\Omega, q$ be continuous convex functions. Then, for the optimality of the arc $\tilde{x}(t)$ in the convex problem $(P C)$, it is sufficient that there exist a pair of absolutely continuous functions $\left\{x^{*}(t), \psi^{*}(t)\right\}, t \in[0,1]$ and a function $\alpha(t) \geq 0$ satisfying a.e. the second order adjoint differential SVICs generated by the given second order differential inclusion (i), (ii) and boundary conditions (iii).

Proof. By definition of the subdifferential of function $\Omega$, we rewrite the second order adjoint differential SVICs (i) in the form:

$$
\begin{aligned}
& \alpha(t) \Omega\left(x(t), x^{\prime}(t), x^{\prime \prime}(t)\right)-\alpha(t) \Omega\left(\tilde{x}(t), \tilde{x}^{\prime}(t) \tilde{x}^{\prime \prime}(t)\right) \\
& \geq\left\langle\frac{d^{2} x^{*}(t)}{d t^{2}}+\frac{d \psi^{*}(t)}{d t}-f_{x}^{\prime}(\tilde{x}(t), t), x(t)-\tilde{x}(t)\right\rangle \\
& +\left\langle\psi^{*}(t), \frac{d(x(t)-\tilde{x}(t))}{d t}\right\rangle-\left\langle x^{*}(t), \frac{d^{2}(x(t)-\tilde{x}(t))}{d t^{2}}\right\rangle .
\end{aligned}
$$

On the other hand, since $f(\cdot, t)$ is convex for all feasible solutions $x(\cdot)$

$$
\left\langle-f_{x}^{\prime}(\tilde{x}(t), t), x(t)-\tilde{x}(t)\right\rangle \geq f(\tilde{x}(t), t)-f(x(t), t),
$$

inequality (5.1) can be reduced to

$$
\begin{aligned}
& \alpha(t) \Omega\left(x(t), x^{\prime}(t), x^{\prime \prime}(t)\right)-\alpha(t) \Omega\left(\tilde{x}(t), \tilde{x}^{\prime}(t) \tilde{x}^{\prime \prime}(t)\right) \\
& \geq\left\langle\frac{d^{2} x^{*}(t)}{d t^{2}}+\frac{d \psi^{*}(t)}{d t}, x(t)-\tilde{x}(t)\right\rangle+f(\tilde{x}(t), t)-f(x(t), t) \\
& +\left\langle\psi^{*}(t), \frac{d(x(t)-\tilde{x}(t))}{d t}\right\rangle-\left\langle x^{*}(t), \frac{d^{2}(x(t)-\tilde{x}(t))}{d t^{2}}\right\rangle .
\end{aligned}
$$

From condition (ii) $\alpha(t) \Omega\left(\tilde{x}(t), \tilde{x}^{\prime}(t), \tilde{x}^{\prime \prime}(t)\right)=0$ and $\alpha(t) \Omega\left(x(t), x^{\prime}(t), x^{\prime \prime}(t)\right) \leq 0(\alpha(t) \geq 0)$ for all feasible solutions $x(\cdot)$, it follows from the last inequality that

$$
\begin{aligned}
& 0 \geq\left\langle\frac{d^{2} x^{*}(t)}{d t^{2}}+\frac{d \psi^{*}(t)}{d t}, x(t)-\tilde{x}(t)\right\rangle+f(\tilde{x}(t), t)-f(x(t), t) \\
& +\left\langle\psi^{*}(t), \frac{d(x(t)-\tilde{x}(t))}{d t}\right\rangle-\left\langle x^{*}(t), \frac{d^{2}(x(t)-\tilde{x}(t))}{d t^{2}}\right\rangle .
\end{aligned}
$$


For convenience, let us rewrite the latter inequality as follows:

$$
\begin{aligned}
& f(x(t), t)-f(\tilde{x}(t), t) \geq\left\langle\frac{d^{2} x^{*}(t)}{d t^{2}}, x(t)-\tilde{x}(t)\right\rangle \\
& -\left\langle\frac{d^{2}(x(t)-\tilde{x}(t))}{d t^{2}}, x^{*}(t)\right\rangle+\frac{d}{d t}\left\langle\psi^{*}(t), x(t)-\tilde{x}(t)\right\rangle .
\end{aligned}
$$

Taking into account that $x(\cdot), \tilde{x}(\cdot)$ are feasible $\left(x(0)=\tilde{x}(0)=v_{0}\right)$ and integrating the inequality (5.2) over $[0,1]$, we have

$$
\begin{aligned}
& \int_{0}^{1}[f(x(t), t)-f(\tilde{x}(t), t)] d t \geq \int_{0}^{1}\left[\left\langle\frac{d^{2} x^{*}(t)}{d t^{2}}, x(t)-\tilde{x}(t)\right\rangle-\left\langle\frac{d^{2}(x(t)-\tilde{x}(t))}{d t^{2}}, x^{*}(t)\right\rangle\right] d t \\
&+\left\langle\psi^{*}(1), x(1)-\tilde{x}(1)\right\rangle-\left\langle\psi^{*}(0), x(0)-\tilde{x}(0)\right\rangle \\
&=\int_{0}^{1}\left[\left\langle\frac{d^{2} x^{*}(t)}{d t^{2}}, x(t)-\tilde{x}(t)\right\rangle-\left\langle\frac{d^{2}(x(t)-\tilde{x}(t))}{d t^{2}}, x^{*}(t)\right\rangle\right] d t+\left\langle\psi^{*}(1), x(1)-\tilde{x}(1)\right\rangle .
\end{aligned}
$$

Denoting the expression in the square parentheses on the right hand side of (5.3) by $W$, we can transform it as follows:

$$
W=\frac{d}{d t}\left\langle\frac{d x^{*}}{d t}, x(t)-\tilde{x}(t)\right\rangle-\frac{d}{d t}\left\langle\frac{d(x(t)-\tilde{x}(t))}{d t}, x^{*}(t)\right\rangle .
$$

Then, it can be easily verified that

$$
\begin{aligned}
\int_{0}^{1} W d t= & \left\langle\frac{d x^{*}(1)}{d t}, x(1)-\tilde{x}(1)\right\rangle-\left\langle\frac{d x^{*}(0)}{d t}, x(0)-\tilde{x}(0)\right\rangle \\
& -\left\langle\frac{d(x(1)-\tilde{x}(1))}{d t}, x^{*}(1)\right\rangle+\left\langle\frac{d(x(0)-\tilde{x}(0))}{d t}, x^{*}(0)\right\rangle .
\end{aligned}
$$

Taking taking account that condition (iii) $x^{*}(1)=0$ and $x(t), \tilde{x}(t)$ are feasible solutions $(x(0)=\tilde{x}(0)=$ $\left.v_{0}, x^{\prime}(0)=\tilde{x}^{\prime}(0)=v_{1}\right)$, the relation in (5.4) can be rewritten as follows:

$$
\int_{0}^{1} W d t=\left\langle\frac{d x^{*}(1)}{d t}, x(1)-\tilde{x}(1)\right\rangle .
$$

Thus, (5.3) and (5.5) imply that

$$
\int_{0}^{1}[f(x(t), t)-f(\tilde{x}(t), t)] d t \geq\left\langle\frac{d x^{*}(1)}{d x}, x(1)-\tilde{x}(1)\right\rangle+\left\langle\psi^{*}(1), x(1)-\tilde{x}(1)\right\rangle .
$$

By the condition (iii) for all feasible $\operatorname{arcs} x(t), t \in[0,1]$, we have

$$
q(x(1))-q(\tilde{x}(1)) \geq-\left\langle\psi^{*}(1)+\frac{d x^{*}(1)}{d t}, x(1)-\tilde{x}(1)\right\rangle .
$$

Finally, adding inequalities (5.6) and (5.7), we conclude that $J[x(x)] \geq J[\tilde{x}(t)], \forall x(t), t \in[0,1]$, i.e., $\tilde{x}(t), t \in[0,1]$ is optimal. This completes the proof.

Below, we prove that if function $\Omega$ in the problem (PC) only depends on $x$ and $x^{\prime}$, then the adjoint inclusion only involves one conjugate variable, i.e., there is no auxiliary adjoint variable $\psi^{*}(t)$ in the second order adjoint differential SVICs.

Corollary 5.2. Suppose that for the problem $(P C) \Omega\left(x, x^{\prime}, x^{\prime \prime}\right) \equiv \Omega_{1}\left(x, x^{\prime \prime}\right)$, i.e., function $\Omega$ does not depend on the second argument $x^{\prime}$ and that the conditions of Theorem (5.1) are satisfied. Then, the sufficient conditions of optimality in Theorem (5.1) consist of the following:

a) $\left(\frac{d^{2} x^{*}(t)}{d t^{2}}-f_{x}^{\prime}(\tilde{x}(t), t),-x^{*}(t)\right) \in \alpha(t) \partial_{\left(x, v_{2}\right)} \Omega_{1}\left(\tilde{x}(t), \tilde{x}^{\prime \prime}(t)\right)$ a.e. $t \in[0,1]$, 
b) $-\frac{d x^{*}(1)}{d t} \in \partial q(\tilde{x}(1)), x^{*}(1)=0$

c) $\alpha(t) \Omega_{1}\left(\tilde{x}(t), \tilde{x}^{\prime \prime}(t)\right)=0, \alpha(t) \geq 0$ a.e. $t \in[0,1]$.

Proof. Obviously, by passing to the formal limit in the conditions of Theorem (4.5), we have the conditions (a)-(c) of the corollary. From the proof of Theorem (5.1), we can prove optimality of the trajectory $\tilde{x}(t)$ immediately.

Let us consider one important particular case of the problem (PC) labelled as (P2):

$$
\begin{aligned}
& \operatorname{minimize} J[x(\cdot)]=\int_{0}^{1} f(x(t), t) d t+q(x(1)), \\
& \Omega_{2}\left(x(t), x^{\prime}(t)\right) \leq 0, \text { a.e. } t \in[0,1], \\
& x(0)=v_{0} .
\end{aligned}
$$

By passing to the limit procedure in the conditions of Theorem (5.11), we have the conditions (d)-(f):

d) $\left(\frac{d u^{*}(t)}{d t}-f_{x}^{\prime}(\tilde{x}(t), t), u^{*}(t)\right) \in \alpha(t) \partial_{\left(x, v_{1}\right)} \Omega_{2}\left(\tilde{x}(x), \tilde{x}^{\prime}(t)\right)$,

e) $-u^{*}(1) \in \partial q(\tilde{x}(1))$,

f) $\alpha(t) \Omega_{2}\left(\tilde{x}(t), \tilde{x}^{\prime}(t)\right)=0, \alpha(t) \geq 0$.

We need to show that the conditions (d)-(f) are sufficient for optimality of $\tilde{x}(t)$.

Theorem 5.3. Suppose that in the problem $(P C) \Omega\left(x, x^{\prime}, x^{\prime \prime}\right) \equiv \Omega_{2}\left(x, x^{\prime}\right)$, i.e., the function $\Omega$ does not depend on the third argument $x^{\prime \prime}$ and that the conditions of Theorem (5.1) are satisfied. Then, for optimality of the trajectory $\tilde{x}(t)$ in the problem (P2), it is sufficient that there exist functions $u^{*}(t)$ and $\alpha(t) \geq 0$ satisfying the conditions $(d)-(f)$.

Proof. Using condition (d), we have

$$
\begin{aligned}
& \alpha(t) \Omega_{2}\left(x(t), x^{\prime}(t)\right)-\alpha(t) \Omega_{2}\left(\tilde{x}(t), \tilde{x}^{\prime}(t)\right) \\
& \geq\left\langle\frac{d u^{*}(t)}{d t}-f_{x}^{\prime}(\tilde{x}(t), t), x(t)-\tilde{x}(t)\right\rangle+\left\langle u^{*}(t), x^{\prime}(t)-\tilde{x}^{\prime}(t)\right\rangle .
\end{aligned}
$$

From (5.2), we find that

$$
f(x(t), t)-f(\tilde{x}(t), t) \geq \frac{d}{d t}\left\langle u^{*}(t), x(t)-\tilde{x}(t)\right\rangle .
$$

Integrating this inequality over the interval $[0,1]$ and taking into account that $x(\cdot)$ and $\tilde{x}(\cdot)$ are feasible $\left(x(0)=\tilde{x}(0)=v_{0}\right)$, we find that

$$
\int_{0}^{1}[f(x(t), t)-f(\tilde{x}(t), t)] d t \geq\left\langle u^{*}(1), x(1)-\tilde{x}(1)\right\rangle .
$$

It follows that

$$
q(x(1))-q(\tilde{x}(1)) \geq-\left\langle u^{*}(1), x(1)-\tilde{x}(1)\right\rangle .
$$

From (5.8) and (5.9), we obtain that $J[x(t)] \geq J[\tilde{x}(t)], \forall x(t) \in[0,1]$, i.e., $\tilde{x}(t), t \in[0,1]$ is optimal. This completes the proof. 
Corollary 5.4. Suppose now that $\Omega$ is a continuously differentiable function. Then, condition (i) of Theorem (5.1) can be simplified as follows:

$$
\begin{aligned}
\frac{d^{2}}{d t^{2}}\left[\alpha(t) \Omega_{v_{2}}^{\prime}\left(\tilde{x}(t), \tilde{x}^{\prime}(t), \tilde{x}^{\prime \prime}(t)\right)\right] & -\frac{d}{d t}\left[\alpha(t) \Omega_{v_{1}}^{\prime}\left(\tilde{x}(t), \tilde{x}^{\prime}(t), \tilde{x}^{\prime \prime}(t)\right)\right] \\
& +\alpha(t) \Omega_{x}^{\prime}\left(\tilde{x}(t), \tilde{x}^{\prime}(t), \tilde{x}^{\prime \prime}(t)\right)+f_{x}^{\prime}(\tilde{x}(t), t)=0 .
\end{aligned}
$$

Proof. Indeed, from the condition

$$
\partial_{\left(x, v_{1}, v_{2}\right)} \Omega\left(\tilde{x}(t), \tilde{x}^{\prime}(t), \tilde{x}^{\prime \prime}(t)\right)=\left\{\Omega_{x}^{\prime}\left(\tilde{x}(t), \tilde{x}^{\prime}(t), \tilde{x}^{\prime \prime}(t)\right), \Omega_{v_{1}}^{\prime}\left(\tilde{x}(t), \tilde{x}^{\prime}(t), \tilde{x}^{\prime \prime}(t)\right), \Omega_{v_{2}}^{\prime}\left(\tilde{x}(t), \tilde{x}^{\prime}(t), \tilde{x}^{\prime \prime}(t)\right)\right\}
$$

and condition (i) of Theorem (5.1), we arrive at

$$
\begin{aligned}
& \frac{d^{2} x^{*}(t)}{d t^{2}}+\frac{d \psi^{*}(t)}{d t}-f_{x}^{\prime}(\tilde{x}(t), t)=\alpha(t) \Omega_{x}^{\prime}\left(\tilde{x}(t), \tilde{x}^{\prime}(t), \tilde{x}^{\prime \prime}(t)\right), \\
& \psi^{*}(t)=\alpha(t) \Omega_{v_{1}}^{\prime}\left(\tilde{x}(t), \tilde{x}^{\prime}(t), \tilde{x}^{\prime \prime}(t)\right), \quad-x^{*}(t)=\alpha(t) \Omega_{v_{2}}^{\prime}\left(\tilde{x}(t), \tilde{x}^{\prime}(t), \tilde{x}^{\prime \prime}(t)\right),
\end{aligned}
$$

which implies the desired result immediately.

Theorem 5.5. Let us consider the nonconvex problem (2.4)- (2.6), i.e., $f(\cdot, t): \mathbb{R}^{n} \rightarrow \mathbb{R}^{1}$ and $\Omega$, q are nonconvex functions. Then, for optimality of the arc $\tilde{x}(t), t \in[0,1]$ in the problem $(P C)$, it is sufficient that there exist a pair of absolutely continuous functions $\left\{x^{*}(t), \psi^{*}(t)\right\}, t \in[0,1]$ and a function $\alpha(t) \geq 0$ satisfying following conditions:

a) $\left(\frac{d^{2} x^{*}(t)}{d t^{2}}+\frac{d \psi^{*}(t)}{d t}-x^{*}(t), \psi^{*}(t),-x^{*}(t)\right) \in \alpha(t) \partial_{\left(x, v_{1}, v_{2}\right)} \Omega\left(\tilde{x}(t), \tilde{x}^{\prime}(t), \tilde{x}^{\prime \prime}(t)\right)$, a.e. $t \in[0,1]$,

b) $f(x, t)-f(\tilde{x}(t), t) \geq\left\langle x^{*}(t), x-\tilde{x}(t)\right\rangle, \forall x \in \mathbb{R}^{n}$,

c) $q(x)-q(\tilde{x}(1)) \geq-\left\langle\psi^{*}(1)+\frac{d x^{*}(1)}{d t}, x-\tilde{x}(1)\right\rangle, \forall x \in \mathbb{R}^{n}, x^{*}(1)=0$,

d) $\alpha(t) \Omega\left(\tilde{x}(t), \tilde{x}^{\prime}(t), \tilde{x}^{\prime \prime}(t)\right)=0, \alpha(t) \geq 0$, a.e. $t \in[0,1]$.

Proof. Using condition (a) in the nonconvex case, one has

$$
\begin{aligned}
& \alpha(t) \Omega\left(x(t), x^{\prime}(t), x^{\prime \prime}(t)\right)-\alpha(t) \Omega\left(\tilde{x}(t), \tilde{x}^{\prime}(t), \tilde{x}^{\prime \prime}(t)\right) \\
& \geq\left\langle\frac{d^{2} x^{*}(t)}{d t^{2}}+\frac{d \psi^{*}(t)}{d t}-x^{*}, x(t)-\tilde{x}(t)\right\rangle \\
& +\left\langle\psi^{*}(t), \frac{d(x(t)-\tilde{x}(t))}{d t}\right\rangle-\left\langle x^{*}(t), \frac{d^{2}(x(t)-\tilde{x}(t))}{d t^{2}}\right\rangle .
\end{aligned}
$$

Using condition (b), (5.2) is justified. Thus, the rest of the proof is similar to the one of Theorem (5.1).

Remark 5.6. We remark that the optimality problems (PD) and (PC) can be investigated for different boundary value conditions. For example, for simplicity, let us consider the problem (PC) with the boundary conditions $x(0) \in \mathrm{Q}_{0}, x(1) \in \mathrm{Q}_{1}$, where $\mathrm{Q}_{0}, \mathrm{Q}_{1} \subset \mathbb{R}^{n}$ are convex subsets. For such boundary value problems with SVICs, it can be shown that condition (iii) of Theorem (5.1) should be replaced by the following conditions

$$
\begin{aligned}
& -\psi^{*}(0)-\frac{d x^{*}(0)}{d t} \in K_{\mathrm{Q}_{0}}^{*}(\tilde{x}(0)), \quad \psi^{*}(1) \in K_{\mathrm{Q}_{1}}^{*}(\tilde{x}(1)), \\
& -\frac{d x^{*}(1)}{d t} \in \partial \phi(\tilde{x}(1)), \quad x^{*}(0)=x^{*}(1)=0 .
\end{aligned}
$$


Using the boundary conditions in (5.10) and condition $x^{*}(0)=x^{*}(1)=0$, it can be justified that for a problem (PC) with SVICs and the boundary conditions $x(0) \in \mathrm{Q}_{0}, x(1) \in \mathrm{Q}_{1}$, the relation in (5.6) has the form

$$
\begin{aligned}
\int_{0}^{1}[f(x(t), t)-f(\tilde{x}(t), t)] d t \geq & \left\langle\psi^{*}(1)+\frac{d x^{*}(1)}{d t}, x(1)-\tilde{x}(1)\right\rangle \\
& -\left\langle\psi^{*}(0)+\frac{d x^{*}(0)}{d t}, x(0)-\tilde{x}(0)\right\rangle .
\end{aligned}
$$

Then, from the condition $x^{* \prime}(1) \in \partial q(\tilde{x}(1))$ or equivalently from the inequality

$$
q(x(1))-q(\tilde{x}(1)) \geq-\left\langle\frac{d x^{*}(1)}{d t}, x(1)-\tilde{x}(1)\right\rangle
$$

and from the relation in (5.11), we can find the desired result. This completes the proof.

Next, we consider the following example.

Example 5.7. The numerical example given below illustrates the feasibility and effectiveness of the obtained theoretic results:

$$
\begin{aligned}
\operatorname{minimize} & x(1), \\
& x(t)-2 x^{\prime}(t) \leq 0, \\
& x(0)=1, t \in[0,1] .
\end{aligned}
$$

According to problem (P2), $\Omega_{2}(x(t), \tilde{x}(t))=x(t)-2 x^{\prime}(t), f(x(t)) \equiv 0, q(x(t))=x(t)$, where $\Omega_{2}: \mathbb{R}^{2} \rightarrow$ $\mathbb{R}^{1}$ is a linear function, i.e., $\Omega_{2}(x, v)=x-2 v$. By conditions (d)-(f), we show that the optimal trajectory is $\tilde{x}(t)=e^{0.5 t}$ and the minimal value of problem (5.12) is $\tilde{x}(t)=e^{0.5}$. First, it can be easily seen that the subdifferential of $\Omega_{2}$ is the gradient vector $\{1,-2\}$, i.e., $\partial_{(x, v)} \Omega_{2}(x, v)=\{1,-2\}$. We also have $\partial q(x)=\{1\}$. Using the sufficient conditions of optimality (d)-(f), we arrive at

d) $\frac{d u^{*}(t)}{d t}=\alpha(t), u^{*}(t)=-2 \alpha(t)$,

e) $u^{*}(1)=-1$,

f) $\alpha(t)\left[\tilde{x}(t)-2 \tilde{x}^{\prime}(t)\right]=0, \alpha(t) \geq 0$.

In view of (d) and (e), we have an initial value problem consisting of a simple linear differential equation with constant coefficients:

$$
\frac{d u^{*}(t)}{d t}+\frac{1}{2} u^{*}(t)=0, \quad u^{*}(1)=-1,
$$

whose solution is $u^{*}(t)=-e^{\frac{1}{2}(1-t)}$. Then,

$$
\frac{d u^{*}(t)}{d t}=\alpha(t)=\frac{1}{2} e^{\frac{1}{2}(1-t)} .
$$

The nonnegative function $\alpha(t)=0.5 e^{0.5(1-t)}$ in (5.13) is nonzero over the entire time interval $[0,1]$. Therefore, by the condition (f), $\tilde{x}(t)-2 \tilde{x}^{\prime}(t)=0$ and the solution of the initial value problem

$$
\tilde{x}(t)-2 \tilde{x}^{\prime}(t)=0, x(0)=1
$$

is $\tilde{x}(t)=e^{0.5 t}$ and its minimum value at the point $t=1$ is $e^{0.5}$. 


\section{CONCLUSION}

This paper presents a new discretization approach to solve the optimization of Bolza problem with second-order differential inequalities which are often used to describe various processes in science and engineering. Based on the proposed discretization method, the problem with the second-order discreteapproximation inequalities are investigated. Equivalence theorems for subdifferential inclusions and adjoint discrete and discrete-approximate inclusions are basic tools in the study of optimality conditions for discrete and discrete-approximate problems. This approach plays an important role in derivation of second-order adjoint discrete and discrete-approximate inequalities. Necessary and sufficient conditions of optimality for such problems are deduced. As an open problem for further investigations, we mention here the study of optimality discrete approximate for higher order and finite number of differential inequalities and the proposed method is reliable for solving various optimization problems with higher order discrete and differential inequalities.

\section{Acknowledgements}

The authors would like to express their sincere thanks to the handling editor and the anonymous referees for their valuable suggestions which helped to improve this paper.

\section{REFERENCES}

[1] R.P. Agarwal, D. O'Regan, S.H. Saker, Oscillation criteria for second-order nonlinear neutral delay dynamic equations, J. Mathe. Anal. Appl. 300 (2004), 203-217.

[2] S.Y. Cho, Generalized mixed equilibrium and fixed point problems in a Banach space, J. Nonlinear Sci. Appl. 9 (2016), 1083-1092.

[3] M.S. Bazaraa, J.J. Goode, M.Z. Nashed, On the cones of tangents with applications to mathematical programming, J. Optim. Theory Appl. 13 (1974), 389-426.

[4] M. Benchohra, J. Henderson, S. Ntouyas, Impulsive Differential Equations and Inclusions, Hindawi, New York, 2006.

[5] F.H. Clarke, Functional Analysis, Calculus of Variations and Optimal Control, Springer, London, 2013.

[6] K.Q. Lan, G.C. Yang, Optimal constants for two point boundary value problems, Discrete Contin. Dyn. Syst. (2007), 624-633.

[7] J. Li, E.A. Ok, Optimal solutions to variational inequalities on Banach lattices, J. Math. Anal. Appl. 388 (2012), 11571165.

[8] X. Qin, S.Y. Cho, L. Wang, Strong convergence of an iterative algorithm involving nonlinear mappings of nonexpansive and accretive type, Optimization, 67 (2018), 1377-1388.

[9] E.N. Mahmudov, Optimization of second order differential inclusions with boundary value conditions, J. Nonlinear Convex Anal. 18 (2017), 1653-1664.

[10] E.N. Mahmudov, Free time optimization of higher order differential inclusions with endpoint constraints, Appl. Anal. 97 (2018), 2071-2084.

[11] M. Bohner, C.C. Tisdell, Second order dynamic inclusions, J. Nonlin. Math. Phys. 12 (2005), 36-45.

[12] V. Lupulescu, Viable solutions for second order nonconvex functional differential inclusions, Electron. J. Differential Equations 2005 (2005), Article ID 110.

[13] E.N. Mahmudov, Approximation and optimization of higher order discrete and differential inclusions, Nonlinear Differ. Equ. Appl. NoDEA 21 (2014), 1-26. 21 (2014), 1-26.

[14] E.N. Mahmudov, Mathematical programming and polyhedral optimization of second order discrete and differential inclusions, Pacific J. Optim. 11 (2015), 495-525.

[15] E.N. Mahmudov, Optimization of Mayer problem with Sturm-Liouville-type differential inclusions, J. Optim. Theory Appl. 177 (2018), 345-375. 
[16] R.V. Vinter, H. Zheng, Necessary conditions for optimal control problems with state constraints, Trans. Amer. Math. Soc. 350 (1998), 181-204.

[17] E.N. Mahmudov, Approximation and Optimization of Discrete and Differential Inclusions, Elsevier, Massachusetts, 2011.

[18] E.N. Mahmudov, Necessary and sufficient conditions for discrete and differential inclusions of elliptic type, J. Math. Anal. Appl. 323 (2006), 768-789.

[19] B.S. Mordukhovich, Variational Analysis and Generalized Differentiation I \& II, Springer, New York, 2006. 